\title{
The Control of Fatty Acid Composition in Glycerolipids
}

\author{
WILLIAM E. M. LANDS and PRISCILLA HART, Department of Biological Chemistry, \\ The University of Michigan, Ann Arbor, Michigan
}

\begin{abstract}
The continued suggestion that the ratio of saturated to unsaturated acids in the diet can influence the general health of a mammal is still not easily explained at a biochemical level. Studies on the metabolism of glycerophosphatides show that the enzymes involved appear to differ in the degree of selectivity toward substrates with different amounts of unsaturation. In general, each individual acid will have its own characteristic metabolic fate. The acyl-CoA :acyl-GPC acyltransferases have a marked specificity for both the position to be acylated and the particular acylCoA involved. In some cases, the observed distributions of fatty acids in naturally occurring lecithins and triglycerides are similar to the distributions predicted from in vitro studies of the acyltransferase enzymes. Other enzymatic steps, such as acid activation and $\mathrm{N}$-methylation, can also be shown to influence the fatty acid composition of the glycerolipids. The relative importance of these metabolic processes must now be determined.
\end{abstract}

\section{Introduction}

$\mathrm{O}$ UR LABORATORY IS NOW considering the role of lipids in several different membranes. We are following clues developed by others suggesting that phospholipids are essential for the functional integrity of certain enzymes located in membranes. In turn, we know that many membrane-bound enzymes are essential for the formation of phospholipids. Therefore, to some degree, the membrane environment may influence the nature of the products, and the products, the environment. With this interdependence in mind I wish to describe some of the metabolic factors in the control of the fatty acid composition of glycerolipids that are now known.

The first and most important fact to consider is that all of the different fatty acids occurring in nature cannot be esterified simultaneously to one glycerol molecule. Thus the concept of a population of lecithins containing distinct species is inevitable.

The term lecithin should be used either as a plural noun or an adjective since it seldom refers to a single entity. This heterogeneity of the lecithin fraction was emphasized by Hanahan in 1960 (1), and the more recent work of Collins (2) clearly indicates the nature of this problem. The countercurrent

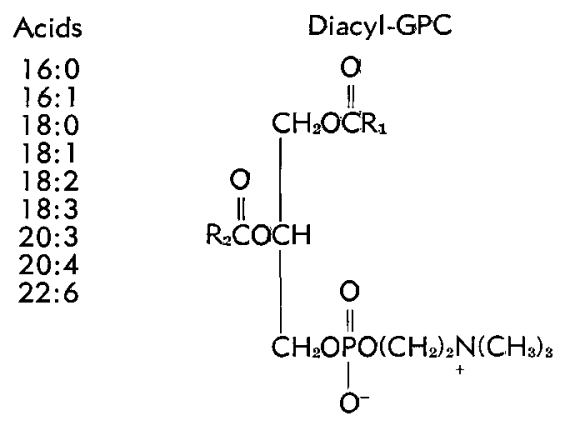

Fig. 1. Lecithin species. separation of the lecithin fraction described by Collins did not give distinct results, but the composition was estimated from data with partial separations. More refined methods have been described by Renkonen (3), and I expect the recent note of Arvidson (4) to provide a stimulating approach to the composition of the lecithins.

Isozaki et al. (5) made a particularly valuable contribution in following the work of Collins (6) and Harris et al. (7) by showing an asymmetric distribution of isotope when ${ }^{14} \mathrm{C}$-methionine was the precursor of lecithins. The result provides a reminder that at least two pathways for lecithin biosynthesis must be considered, and that the different species of lecithins may originate via different pathways.

In our approach to determining the composition of lecithins, we modified the method that was used for other purposes by Tattrie (8) and Hanahan et al. (9). We converted the lecithins to 1,2-diglycerides using $\mathrm{C}$. welchii phospholipase $\mathrm{C}$, and then acylated them with heptadecanoyl anhydride to form triglycerides. The conditions for the latter reaction were described by Mattson et al. (10).

\section{Results and Discussion}

Table I shows the fatty acid composition at each stage of the procedure, indicating no appreciable loss of any specific acids.

The triglycerides were separated according to their alkene content by using thin-layer chromatography on silicic acid impregnated with silver nitrate. Figure 2 shows the quality of separation obtained and also the method for calculating the composition of each band. The triglycerides extracted from each band were converted to methyl esters and analyzed by gas-liquid chromatography with an internal standard of methyl pentadecanoate (15:0). Of course, each mole of triglyceride also contained one equivalent of 17:0 in it. These two standards make the overall quantitation easy to achieve and provide checks in determining the validity of our assignments of species. The sample calculations shown in Figure 2 are for the upper half of the triglyceride band 2 . Note that the sum of $16: 0+18: 0$ equals that for $18: 2$ indicating that these triglycerides are from the saturated-diene, 02 , species of the lecithins. A small amount of the monoene-monoene, 11, species also appears to be present in this band.

TABLE I

Analysis of Rat Liver Lecithins *

\begin{tabular}{|c|c|c|c|c|}
\hline & Lecithins & \multicolumn{2}{|c|}{ Diglycerides Triglycerides } & $\begin{array}{c}\text { Recombined } \\
\text { data }\end{array}$ \\
\hline $\begin{array}{l}16: 0 \\
18: 0 \\
16: 1 \\
18: 1 \\
18: 2 \\
18: 3 \\
20: 3 \\
20: 4 \\
22: 6\end{array}$ & 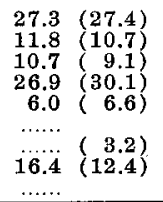 & $\begin{array}{r}25.7 \\
11.6 \\
11.0 \\
26.7 \\
6.9\end{array}$ & $\begin{array}{r}29.6 \\
10.6 \\
10.4 \\
25.3 \\
5.7 \\
0.3 \\
2.5 \\
15.6\end{array}$ & $\begin{aligned} 23.8 \\
12.5 \\
10.7 \\
25.5 \\
8.0 \\
0.7 \\
2.5 \\
13.3 \\
2.6\end{aligned}$ \\
\hline \multicolumn{5}{|c|}{$\begin{array}{l}\text { The percentage composition of each fraction was determined as } \\
\text { described in the experimental section. The reference acids, 15:0 and } \\
\text { 17:0, were omitted for these calculations. The values in parentheses } \\
\text { are for the lecithing from another rat to indicate the variance that } \\
\text { is found for individual animals. The recombined data represent the } \\
\text { sum of the contents of all the resolved bands (see Fig. 2). }\end{array}$} \\
\hline
\end{tabular}




\begin{tabular}{|c|c|c|c|c|c|c|}
\hline \multirow{2}{*}{$\underbrace{0}_{1}$} & \multicolumn{6}{|c|}{ BAND 2A } \\
\hline & Acid & $t$ & H & $\mathrm{t} \times \mathrm{H}$ & RATIO ( ${ }_{M W}$ ) & $\mathrm{m} \mu \mathrm{mole}$ \\
\hline & $15: 0$ & 7.6 & 13.3 & 101.1 & $1.00(1.000)$ & 145 \\
\hline & $17: 0$ & 13.7 & 18.6 & 254.8 & $2.52(.901)$ & 329 \\
\hline & $16: 0$ & 10.2 & 11.9 & 121.4 & $1.20(.948)$ & 165 \\
\hline & $18: 0$ & 18.4 & 5.7 & 104.9 & $1.04(.859)$ & 129 \\
\hline & $18: 1$ & 21.3 & 1.4 & 29.8 & $.29(.865)$ & 37 \\
\hline & $18: 2$ & 26.8 & 8.6 & 230.5 & 2. $28(.871)$ & 289 \\
\hline
\end{tabular}

FIg. 2. Separation of lecithin derivatives. The diagram at the left indicates the separation of bands on the thin-layer plate. The origin is at the lower dotted line, and the solvent front is marked " $F$ ". The numbers indicate the average number of double bonds per triglyceride molecule. The calculations shown at the right are deseribed in the experimental section.

The analytical results for all bands from normal male rat liver lecithins are shown in Figure 3. Note that band 4 contains equal amounts of saturated and dieneoic acids, and represents the 02 species. Apparently only small amounts of the 00 -type lecithins occur in this tissue, and the major species are 04 , 02 and 01 . The data from the different bands permit the calculation of the composition of the original lecithin fraction, the results with three different males being shown in Table II. Although some variance is shown between individual animals, the general pattern is quite apparent. You can see that nearly twothirds of the liver lecithins from normal male rats are the 02 and 04 species.

The separation of the triglycerides derived from liver lecithins of the normal female rat is shown in Figure 4. The pattern is, in general, similar to that found for the male, and the average compositions for the two sexes are compared in Figure 5. The content of the 04 species was higher, and that of the 02 species slightly lower in the female. In addition, the males appeared to have more lecithin species that contained monoenoic acids (presumably at the 1position) with another unsaturated acid.

The differences between the lecithins of the two sexes were slight, and we have no clear idea of how the individual species will influence membrane struc-
$\left(\begin{array}{l}0 \\ 0\end{array}\right)\left(\begin{array}{ll}0 \\ 0\end{array}\right)$

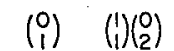
$\left(\begin{array}{l}1 \\ 2\end{array}\right)$
$\left(\begin{array}{l}0 \\ 3\end{array}\right)$
$\left(\begin{array}{l}0 \\ 4\end{array}\right)$
$\left(\begin{array}{l}2 \\ 4\end{array}\right)\left(\begin{array}{l}0 \\ 6\end{array}\right)$

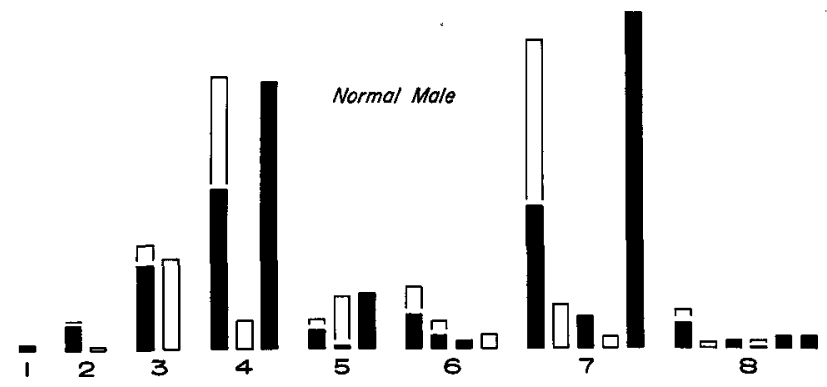

FIG. 3. Liver lecithins from the normal male rat. The fatty acid content (in mumoles) of each band is indicated by the height of the vertical bars. The saturated acids are represented with the solid, lower portion as 16:0, and the open, upper portion as 18:0. The monoenoates also have the solid, lower portion as $16: 1$, and the open, upper portion as $18: 1$. The dienoate, $18: 2$, is shown as a solid bar. The trienoates are shown with the solid, lower portion as $18: 3$, and the open, upper portions as $20: 3$. The tetraenoate, $20: 4$, and hexaenoate, $22: 6$, are shown as solid bars. The numbers in parentheses at the top of the figure indicate the predominant lecithin species represented in the band.
TABLE II

Rat Liver Lecithins a

\begin{tabular}{lrrrr}
\hline \hline \multirow{2}{*}{$\begin{array}{l}\text { Lecithin } \\
\text { species }\end{array}$} & \multicolumn{4}{c}{ Mole \% } \\
\cline { 2 - 5 } & \multicolumn{4}{c}{ Individuals } \\
\hline 00 & 1.8 & 1.1 & $\mathbf{2 . 4}$ & Average \\
01 & $\mathbf{7 . 3}$ & $\mathbf{1 3 . 2}$ & $\mathbf{1 3 . 8}$ & $\mathbf{1 1 . 8}$ \\
11 & 2.3 & 1.1 & 2.5 & 2.0 \\
02 & 24.6 & 27.6 & 31.7 & 28.0 \\
12 & 6.5 & 8.1 & $\mathbf{4 . 9}$ & 2.5 \\
03 & 2.1 & 1.8 & 2.3 & 2.1 \\
13 & 1.0 & 3.7 & 1.2 & 2.0 \\
04 & 38.6 & $\mathbf{3 1 . 6}$ & $\mathbf{3 4 . 6}$ & 34.9 \\
14 & 8.6 & $\mathbf{2 . 3}$ & $\mathbf{4 . 5}$ \\
06 & 6.1 & 7.7 & $\mathbf{1 . 4}$ & $\mathbf{5 . 1}$ \\
\hline
\end{tabular}

a The number of m $\mu$ moles of each species was calculated by adding the amounts in each of the different bands from the thin-layer plates. These values were then converted to mole percentages.

ture and function. Different amounts of lecithins, however, can be correlated with permeability as shown by Van Deenen and his co-workers (11). Other studies have shown that variations in the proportions of different acids in the diet might also affect the stability of erythrocyte and mitochondrial membranes $(12,13)$. These compositional differences must be reflected in a different "population" of lecithins in the membranes. We have found that erythrocyte membranes contain lecithins with fatty acids distributed between the 1- and 2- positions in a manner similar to that found in other tissues. A careful study by Dr. K. Waku in our laboratory of the acyl-CoA :phospholipid acyltransferase activity in the washed erythrocyte "ghosts" showed good activity with 1-acyl-GPC as a substrate. However, no measurable activity was found with 2-acyl-GPC using the same spectrophotometric assay conditions. The acid: $\mathrm{CoA}$ ligase activity was also too low to measure in the "ghosts," indicating a limited metabolic activity for these membranes. These studies emphasize the fact that some of the intermediates in the biosynthesis of lipids must be produced by the soluble enzymes of a cell. The rates of some reactions in the membranes may then be controlled by the supply of those intermediates as substrates for the membrane enzymes. For instance, the relatively high levels of palmitate at the 2-position of erythrocyte lecithins suggests that these cells may have had a disproportionately high supply of this acid as substrate.

Recently Privett and his colleagues have collaborated with me on a study of dietary fatty acids and their fate in rat liver lipids (14). We chose some

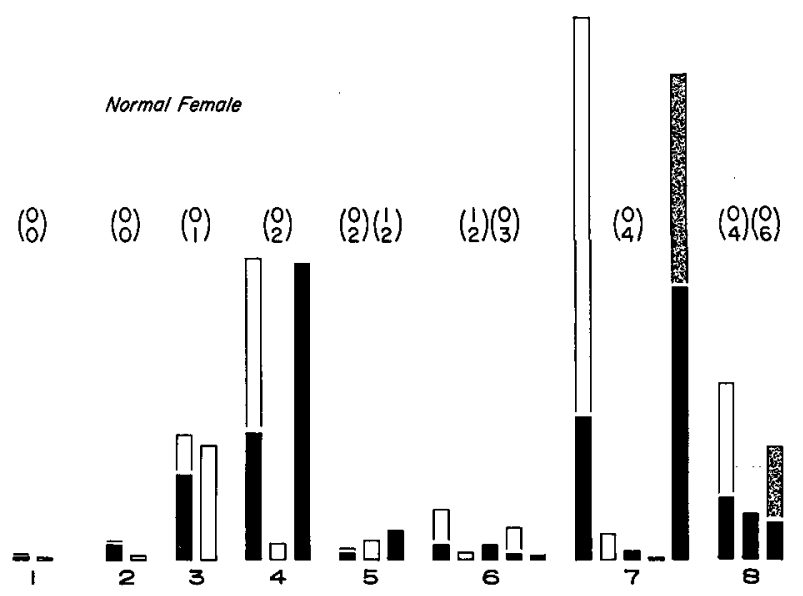

FIG. 4. Liver lecithins from the normal female rat. The data are presented in a similar fashion as in Figure 3 . The stippled areas for $20: 4$ and $22: 6$ represent corrections for the amount of polyunsaturated acid lost in the final stages due to insufficient antioxidant. The correction allows the content of 17:0 to equal one-third of the total acids in that band. 


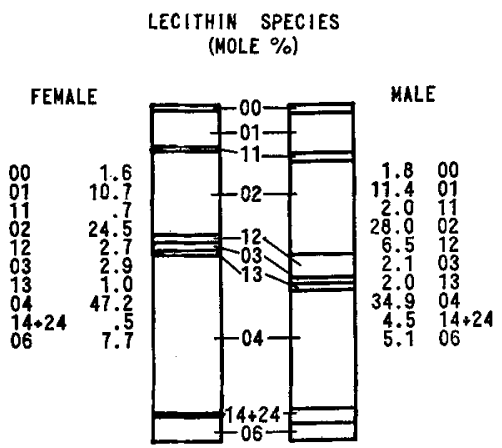

Ftg. 5. Comparison of normal female and male liver lecithins. The average compositions were calculated as in Table II. The values and bar graph at the left are for the data from female rats and those at the right, for males.

unnatural isomers of octadecadienoate that react at different rates with the liver acyltransferases.

A comparison of the behavior of these acids with that of the various naturally occurring acids (see Table III) shows that each one is handled differently by the liver acyltransferases. Rats were fed fat-free diets supplemented with an isomer of octadecadienoate, and the liver lipids were isolated and hydrolyzed to determine the location of the various acids within the lipids. The results in Table IV show that the nature of the supplemental acid did not greatly affect the distribution of palmitate and stearate. Furthermore, the distribution ratios found in the lipids were very close to those observed in the in vitro enzymatic studies. In general, the distribution ratios of all acids measured had a predictable relationship to the values obtained in the acyltransferase assays.

Another phenomenon noted in this work is the similarity in the $2 / 1$ distribution ratio for an acid in triglycerides and that in the leeithins. Such a result suggests that the liver triglyceride composition might be controlled in part by the specificity of the acyl-CoA :phospholipid acyltransferases. This suggestion is also supported by the results in Table V which compare our measurements of the specificity of pig liver acyltransferases (15) with the triglyceride compositions reported by Barford et al. (16).

In considering the metabolic processes that could produce these patterns in the glycerolipids, we felt that a selective synthesis of phosphatidic acids from glycerol phosphate was an obvious point of metabolic control.

We have found that two types of enzymes are involved in synthesizing phosphatidic acids (15). The enzyme that catalyzes the esterification of the first fatty acid with glycerol phosphate apparently contains a mercaptan group essential for its catalytic

TABLE III

Rat Liver Acyltransferase Activities a

\begin{tabular}{|c|c|c|c|}
\hline \multirow{2}{*}{ Acyl-CoA } & \multicolumn{2}{|c|}{ Position acylated } & \multirow{2}{*}{ 2/1 Ratio } \\
\hline & No. 2 & No. 1 & \\
\hline $\begin{array}{l}18: 0 \\
16: 0 \\
18: 1 \mathrm{c} \\
18: 1 \mathrm{t} \\
18: 2 \mathrm{cc} \\
18: 2 \mathrm{ct} \\
18: 2 \mathrm{te} \\
18: 2 \mathrm{tt} \\
18: 3 \mathrm{ecc} \\
20: 4 \mathrm{cccc}\end{array}$ & $\begin{array}{r}1.7 \\
3.5 \\
12.4 \\
10.3 \\
23.1 \\
9.1 \\
13.7 \\
6.1 \\
14.6 \\
19.6\end{array}$ & $\begin{array}{r}19.6 \\
20.3 \\
3.9 \\
23.3 \\
1.8 \\
2.0 \\
13.8 \\
\mathbf{2 8 . 4} \\
1.8 \\
\mathbf{2 . 3}\end{array}$ & $\begin{array}{c}0.087 \\
0.17 \\
3.2 \\
0.44 \\
12.9 \\
4.6 \\
1.0 \\
0.21 \\
8.0 \\
8.5\end{array}$ \\
\hline
\end{tabular}

TABLE IV

Composition of Rat Liver Lipids a

\begin{tabular}{|c|c|c|c|}
\hline \multirow{2}{*}{$\begin{array}{l}\text { Dietary } \\
\text { supplements }\end{array}$} & \multirow{2}{*}{$\begin{array}{l}\text { Lipid } \\
\text { tested }\end{array}$} & \multicolumn{2}{|c|}{ 2/1 Ratio } \\
\hline & & $18: 0$ & $16: 0$ \\
\hline $18: 2 \mathrm{cc}$ & Lecithins & 0.10 & 0.12 \\
\hline & Triglycerides & .087 & .13 \\
\hline $18: 2 \mathrm{ct}$ & Lecithins & .059 & .11 \\
\hline & Triglycerides & & .12 \\
\hline $18: 2 \mathrm{tt}$ & $\begin{array}{l}\text { Lecithins } \\
\text { Triglycerides }\end{array}$ & .088 & 13 \\
\hline \multicolumn{2}{|c|}{ Acyltransferase rates } & 087 & 17 \\
\hline
\end{tabular}

action. The second enzyme, converting acyl glycerol phosphate to diacylglycerol phosphate, resembled the other acyltransferases in being relatively unaffected by reagents that react with mercaptans. The second enzyme is particularly sensitive, however, to inhibition by its substrate, acyl-CoA. Such an inhibition by this highly charged, anionic detergent makes it difficult to do the usual kinetic studies. One of our approaches to defining the over-all positional specificity of the two enzymes used ${ }^{14}$ C-labeled acids, with the result that the over-all pathway in guinea pig liver microsomes appeared to be nearly random process (17). Later studies of the acyl-CoA :acylglycerol phosphate acyltransferase gave a ratio of $20 / 30$ for esterification rates of $18: 0$ and 18:2 respectively at the 2-position (15). If we assume, for the moment, that the first enzyme catalyzed the esterification of the two acids randomly, we could predict that $44 \%$ of the incorporated stearate and $54 \%$ of the incorporated linoleate would be at the 2-position. The observed results were $40 \%$ and $52 \%$, respectively. Thus, we still have not demonstrated a high degree of specificity for these enzymes in guinea pig liver. Perhaps the biosynthesis of phosphatidic acid may not be selective enough to produce the distribution patterns of fatty acids reported for the glycerolipids in vivo.

Returning to the question of the role of phospholipids in membranes, we wanted to investigate further into systems where the membrane "integrity" has been altered. We felt that the results of several workers show that starving and refeeding will alter the levels of many enzymes in liver. The ones of special interest to us are those locked tightly in the membranes of the endoplasmic reticulum. Repeated unsuccessful attempts to purify these enzymes lead to an impression that they are integral units of the normal membrane. In this way, the specific activity of an enzyme, e.g., glucose-6-phosphatase, can become a characteristic property of the unimpaired membrane. A rise and fall in the activity of these enzymes in the cell must indicate either an altered "integrity" of the membrane, or an increased synthesis and destruction of the membrane itself. If, as we now believe, the phospholipids are intimately involved in maintaining the functional qualities of these membranes, a clearer knowledge of the composition of the lecithin species could be helpful in understanding this role.

TABLE V

Distribution Ratios Between the Secondary and a Primary Position in Pig Liver Lipids

\begin{tabular}{lrrrr}
\hline & $16: 0$ & $18: 0$ & $18: 1$ & $18: 2$ \\
\hline Triglycerides (16) & 0.3 & 0.08 & 1.8 & 1.9 \\
Acyltransferase (15) & 0.2 & 0.15 & 1.9 & 1.8 \\
\hline
\end{tabular}




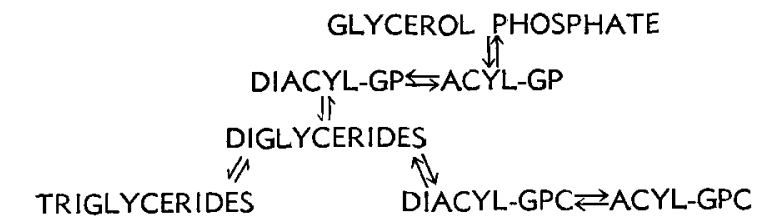

FIG. 6. Metabolie relationship between triglycerides and lecithins.

An analysis of the liver lecithin species from rats that had been starved and then fed a fat-free diet is shown in Figure 7 . The acids of band 4 indicate that there is a very large amount of the 11 species which was almost undetectable in the normal males. There was a marked decrease in the 02 and the dominant species became 01 and 11 in addition to 04 . We also noted a slight change in the appearance of the microsomal fractions from these livers. The shifts in phospholipid species brought on by this dietary change are summarized in Table VI. The lecithins from the starved animals did not differ appreciably from those in the normal, but the results with refed animals show a large decrease in the 02 and 04 species. This results supports the comments of Allman et al. (18) who indicated that such a diet causes a local essential fatty acid deficiency in the liver. Preliminary experiments in our laboratory with livers from rats with a prolonged essential fatty acid deficiency showed a similar pattern to that in the refed animals with 20:3 replacing 18:2 and 20:4. Refed female rats gave results similar to those for the males, although the 01 species was elevated much more than the 11 type (see Fig. 8). The summary in Table VII shows that, in general, the female rats have a lower tendency to form lecithins with two unsaturated acids than the males.

The wide range in the observed amounts of a given lecithin species in a certain tissue tempted us to test again whether these patterns could have been predicted from what we already know of lecithin metabolism. To do this, we used the average fatty acid content of the lecithin fraction and the $2 / 1$ ratios for the rates of enzyme-catalyzed esterification. With these two numbers, we predicted the pattern of species that would result if no other enzymatic specificities were involved. The values given in Table VIII also include those calculated on the basis of a fully random esterification of the component acids without regard for the specificities of acyl-CoA: phospholipid acyltransferase. The results emphasize one thing that is well known-the acids are not distributed within the lecithins in a fully random

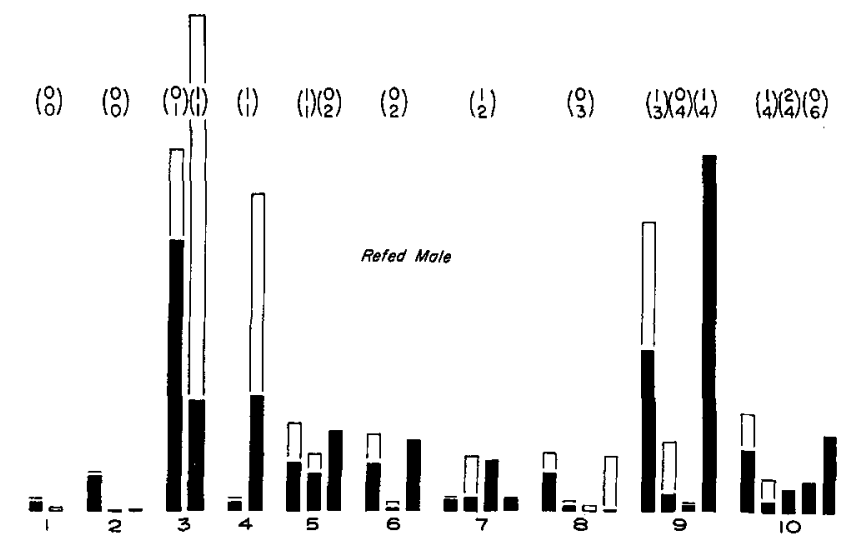

FIG. 7. Liver lecithins from starved and refed male rats. The data are presented in a similar fashion as in Figure 3.

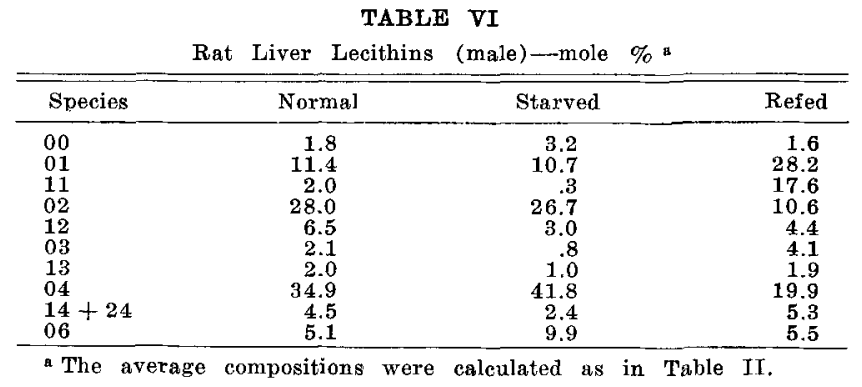

fashion. The speeific activity of the acyltransferases was not altered by the changes in the diet and the relative rates of esterification of the different acids were similar in normal, starved and refed rats. Therefore, although other enzyme activities rise and fall, the acyltransferases appear to be a more stable constituent of the liver cell. The close fit of the observed pattern of lecithin species with that predicted on the basis of normal acyltransferase rates, suggests that two features are dominant in controlling the composition of glycerolipids in vivo, the supply of the acid and the specificities of the acyltransferases.

Although we are pleased that the predicted pattern of lecithin species was fairly close to that found in the tissue, one phenomenon cannot be easily explained by what we now know of lecithin metabolism. A review of Figures $2,3,5$ and 6 shows different relative amounts of 16:0 and 18:0 in the lecithin species with differing degrees of unsaturation. This difference was noted by nearly every previous investigator of lecithin heterogeneity; our own results are summarized in Figure 9. These results point out again that each fatty acid must be regarded as having its own metabolic fate, and even the different saturated acids are not treated in the same fashion. Although we have tried several different types of experiment to determine the enzymatic factors controlling the differential esterification of $16: 0$ and $18: 0$ in the glycerolipids, we have not yet identified a definite factor. Thus you can see that today we have a considerable ability to predict the species of lecithins that will occur in a given "population" from rat liver, but several intriguing problems remain to be discussed on another day.

\section{Experimental}

Isolation of Liver Lecithins

Approximately $1.0 \mathrm{~g}$ of tissue was extracted by homogenizing with $4 \mathrm{ml}$ of methanol-chloroform $(2: 1)$ in a Virtis microhomogenizer for $2 \mathrm{~min}$. A small
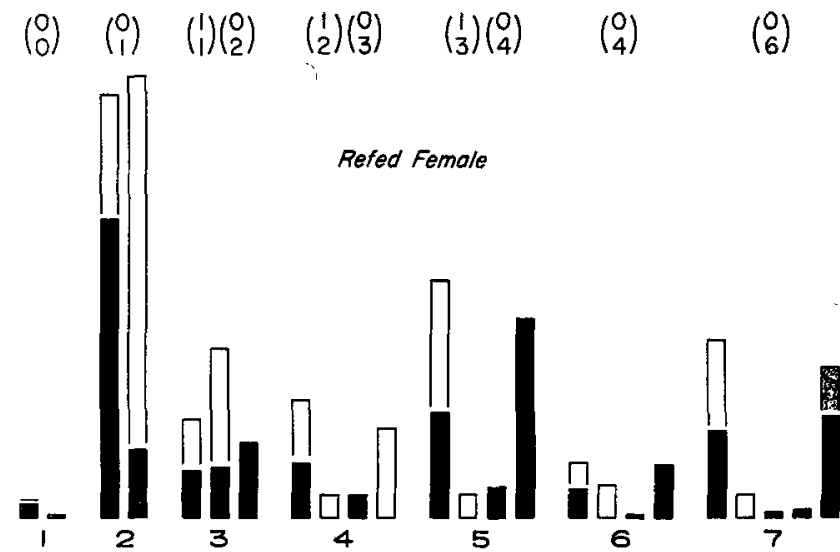

FIG. 8. Liver lecithins from starved and refed female rats. The data are presented in a similar fashion as in Figure 4 . 
TABLE VII

Rat Liver Lecithins (female)--mole \% a

\begin{tabular}{lrrr}
\hline \hline Species & Normal & Starved & Refed \\
\hline 00 & 1.8 & 3.2 & 1.6 \\
01 & 10.7 & 7.3 & 40.2 \\
11 & 24.5 & 26.3 & 11.3 \\
02 & 2.7 & 3.6 & 7.3 \\
12 & 2.9 & 0 & 1.6 \\
03 & 1.0 & 42.0 & 5.3 \\
13 & 47.2 & 1.8 & .8 \\
04 & .5 & 17.0 & .7 \\
$14+24$ & 7.7 & & .4 \\
06 & & &
\end{tabular}

amount of 1,2-dihydro-6-ethoxy-2,2,4-trimethylquinoline, Santoquin, (not more than $100 \mu \mathrm{l}$ of a $1 \%$ solution in benzene) was added to the homogenate and to each subsequent lipid fraction isolated in the following procedure. Four milliliters of chloroform were added and homogenization continued for $2 \mathrm{~min}$. Finally, $1 \mathrm{ml}$ of water was added and the tissue homogenized for another 30 sec. After centrifugation, the clear upper aqueous layer was carefully removed with a pipet and discarded. The organic layer was then removed from beneath the interfacial layer of protein. The protein was rinsed with $5 \mathrm{ml}$ of methanol, and the methanol was added to the original organic layer.

The organic layer was evaporated, the lipid dissolved in diethyl ether-ethanol $(9: 1)$ and placed on a $4 \mathrm{~g}$ column of silicic acid suspended in the same solvent. Neutral lipids and cephalins were eluted with $25 \mathrm{ml}$ of diethyl ether-ethanol $(9: 1)$ and 50 $\mathrm{ml}$ of diethyl ether-ethanol (1:1). The amount of 25 $\mathrm{ml}$ of ethanol-methanol $(9: 1)$ was then used to remove any remaining acidic glycerolipids. The lecithins were then eluted with $50 \mathrm{ml}$ of methanol. A small aliquot portion (4\%) was removed for analysis of the component acids.

\section{Formation of 1,2-Diglycerides}

The remaining lecithin solution was evaporated, and the lipid transferred to a $20 \mathrm{ml}$ screw-cap vial. The lipid was then dissolved in $2 \mathrm{ml}$ of diethyl ether. Four microliters of $\mathrm{M}$ calcium chloride and $2 \mathrm{ml}$ of imidazole buffer $(0.2 \mathrm{M}, \mathrm{pH} 7.4)$ containing about $2 \mathrm{mg}$ of $\mathrm{Cl}^{\circ}$ stridium welchii phospholipase $\mathrm{C}$ (Nutritional Biochemicals) were added and the vial shaken vigorously. The reaction was allowed to continue for 2 $\mathrm{hr}$ with intermittent shaking, after which the volume of diethyl ether was increased to about $10 \mathrm{ml}$ and the reaction mixture washed three times with water.

The diethyl ether solution was dried and the lipid quantitatively transferred to a thin-layer chromatographic plate spread with silicic acid (Adsorbosil2, Applied Science Laboratories). The plate was developed in diethyl ether for $5 \mathrm{~cm}$, dried well and then developed in petroleum ether-diethyl ether (3:1) to a

TABLE VIII

Liver Lecithin Species (male) *

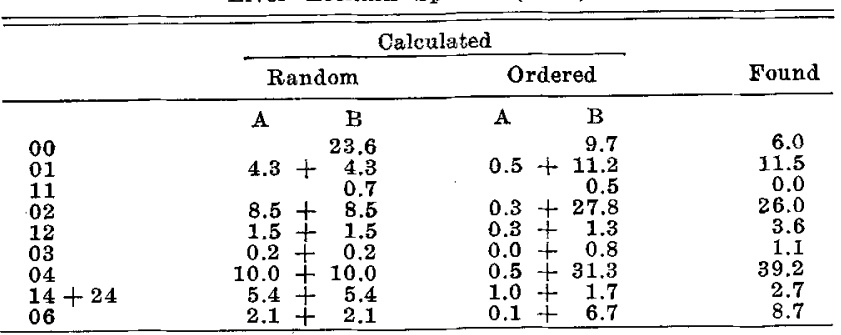

The values in the $A$ columns indicate the amount predicted with the more saturated acid at the 2. Those in the $B$ columns are for the more saturated acid at the 1.
MOLE FRACTION OF $18: 0$
$(0.16-0.59)$

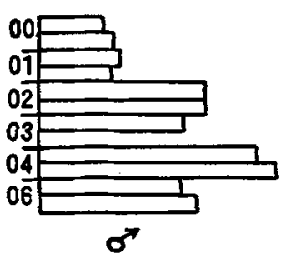

$(0.20-0.74)$

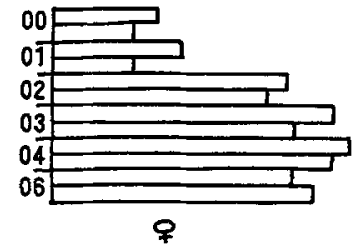

Fig. 9. Mole fraction of 18:0. The content was ealculated in terms of the ratio $18: 6 / 16: 0+18: 0$. The bar graphs indicate the magnitude of this ratio for different bands on the thin-layer plates which correspond to the species indicated on the left of the figure. The range of values obtained for the females and males, respectively, are shown at the top of the figure,

height of $18 \mathrm{~cm}$. The diglyceride region, detected by spraying with dichlorofluoroscein, was scraped free and eluted with diethyl ether containing $5 \%$ methanol. Again a small portion (4\%) was removed for analysis of the component acids of this fraction.

\section{Acylation of Diglycerides}

The diglycerides were transferred to a $9 \times 75 \mathrm{~mm}$ test tube and carefully dried under nitrogen. A solution $(100 \mu \mathrm{l})$ of heptadecanoyl anhydride in benzene $(12 \mathrm{mg} / \mathrm{ml})$ and $0.5 \mu \mathrm{l}$ of $70 \%$ perchloric acid were added. The tube was stoppered, warmed slightly, and allowed to stand at room temperature for 10 min. The reaction mixture was then rinsed into heavy-walled tubes $(12 \times 125 \mathrm{~mm})$ with about $5 \mathrm{ml}$ of benzene. The solution was washed twice with $1.5 \mathrm{ml}$ of water to remove the perchloric acid. The benzene layer was evaporated and the residue transferred to a thin-layer chromatographic plate (Adsorbosil-2). The plate was developed in diethyl ether for $5 \mathrm{~cm}$, dried well, and then developed in petroleum etherdiethyl ether $(9: 1)$ to a height of $18 \mathrm{~cm}$. The triglyceride region, detected by dichlorofluoroscein, was scraped from the plate and eluted with diethyl ether containing 5\% methanol. Again a small portion was removed for analysis of the component acids of this fraction.

\section{Separation of Triglyceride Species}

The triglyceride fraction was transferred to a thinlayer chromatographic plate spread with silicic acid (Silica Gel G, Merck) containing 17\% silver nitrate. To avoid overloading, the sample was applied along the origin line at density of about $1 \mu \mathrm{mole} / \mathrm{cm}$. The plate was developed in petroleum ether-diethyl etherbenzene $(80: 15: 20)$, the triglyceride bands were located with dichlorofluoroscein, and eluted with diethyl ether.

\section{Analysis of Fatty Acids in Lipid Fractions}

One hundred forty-five mumole of methyl pentadecanoate was added to each lipid fraction, and the solvent removed by evaporation under nitrogen. Two milliliters of sodium methoxide $(0.5 \mathrm{M})$ in methanol was added, the mixture shaken, and allowed to stand at room temperature for $10 \mathrm{~min}$. Aqueous hydrochloric acid $(0.2 \mathrm{ml}$ of $6.0 \mathrm{~N})$ was added, followed by $4 \mathrm{ml}$ of petroleum ether and $4 \mathrm{ml}$ of water. The upper organic layer was removed, dried over $\mathrm{NaHCO}_{3}: \mathrm{Na}_{2} \mathrm{SO}_{4}(1: 2)$, and evaporated to dryness. The methyl esters were analyzed by gas-liquid chromatography. 
Gas chromatographic separations were made using $6 \mathrm{ft}$ columns packed with $11 \%$ ethyleneglycol succinate on Gas-Chrom P (Applied Science Laboratories, Inc.) maintained at $190 \mathrm{C}$ with a $40 \mathrm{ml} / \mathrm{min}$ flow of argon. The relative areas were calculated as the product of the retention time and the height of the peak. Each value was then corrected using a factor for the relative molecular weights of methyl pentadecanoate and the acid being measured. The results were then converted to mumoles of esters knowing the amount of 15:0 serving as the internal standard in each chromatographic analysis.

ACKNOWLEDGMENT

This work was supported in part by grant AM-05310 from the USPHS.

\section{REFERENCES}

1. Hanahan, D. J., "Lipid Chemistry," John Wiley and Sons, New York, 1960, p. '77.
2. Collins, F. D., Biochem. J. 88, 319 (1963).

4. Arvidson, G. A. E., J. Lipid Res. 6, 574 (1965).

5. Isozaki, M., A. Yamamoto, T. Amako, Y. Sakai and H. Okita, Med. J. Osaka Univ. 12, 285 (1962)

6. Collins, F. D., Nature 186, 366 (1960).

7. Harris, P. M., D. S. Robinson, and G. S. Getz, Nature 188 742 (1960).

8. Tattrieg, N. H., J. Lipid Res, 1, 60 (1959).

9. Hanahan, D. J., H. Brockerhoff, and E. J. Barron, J. Biol. Chem. 235, 1917 (1960).

10. Mattson, F. H. R. A. Volpenhein, and J. B. Martin, J. Lipid Res. 5,374 (1964).

11. DeGier, J., and L. L. M. Van Deenen, Biochim. Biophys. Acta 84,294 (1964).

12. Walker, B. I., and F. A. Kummerow, Proc. Soc. Exp. Biol Med. $115,1099(1964)$.

13. Stein, O., and Y. Stein, Biochim. Biophys. Acta 84, 621 (1964). 14. Lands, W. E. M., M. L. Blank, L. J. Nutter, and O. S. 14. Lands, W. E. M., M. L. Blank, L. J. Nutter, and O. S.

15. Lands, W. E. M., and P. Hart, J. Biol. Chem. 240, 1905

16. Barford, R. A., F. E. Luddy, S. F. Herb, P. Magidman and R. Wiemenschneider, JAOCS 42,446 (1965)

17. Lands, W. E. M., and P. Hart, J. Lipid Res. 5, 81 (1964)

18. Allman, D. W., D. D. Hubbard, and D. M. Gibson, J. Lipid Res. $6,63(1965)$. 